\title{
On the Dependence of Delta-k Efficiency on Multilooking
}

\author{
Francesco De Zan, Pau Prats-Iraola, Marc Rodriguez-Cassola
}

\begin{abstract}
This paper discusses some aspects of the implementation of Delta-k methods for shift estimation with SAR images. In particular it shows that a common Delta-k algorithm, which postpones the multilooking to the differential interferogram and is therefore robust to the presence of interferometric fringes in the averaging window, does not reach the maximum possible performance and should be better considered as a variant of incoherent cross-correlation. A small adaptation, retaining some multilooking at interferogram level, can significantly improve the efficiency.
\end{abstract}

Index Terms-Synthetic Aperture Radar, SAR interferometry, delay estimation, Delta-k

\section{INTRODUCTION}

Delta-k methods for shift estimation have been introduced into the SAR world by the works of Madsen and Zebker [1]. Several researchers have subsequently reproposed and extended them in different directions. In particular, Scheiber and Moreira in [2] have described an implementation for both range and azimuth shift estimation, being the original Delta-k limited to range signals. In this paper we will talk generically about shift estimation, without the need to make an explicit distinction between range and azimuth directions.

Delta-k algorithms have been applied to precise SAR image coregistration, estimation of shifts for geophysical applications and similar. The related technique called multi-squint has been used for baseline correction in airborne SAR interferometry [3], [4], synchronism recovery in bistatic SAR systems [5] and atmospheric phase screen estimation in SAR interferometry [6].

In their essence, Delta-k methods are based on the fundamental equivalence between delays in time domain and the corresponding phase ramps in the frequency domain: timedomain shifts can thus be estimated as phase differences between different subbands. For distributed scenes two interferograms are generated, one for the lower subband and one for the higher subband, and their phases are differenciated.

In this paper we deal with the performance of two different Delta-k implementations and we show that the "latemultilooking" approach, even though it has some desirable properties, fails to reach the theoretical performance bound. We propose a compromise solution, with a two-stage averaging approach.

\section{General CONSIDERATIONS ON DELTA-K METhods}

Delta-k methods constitute an alternative to time-domain cross-correlation methods [7] with some implementation ad-

The authors are with the DLR (German Aerospace Center), Oberpfaffenhofen, D-82230 Wessling, Germany. e-mail:francesco.dezan@dlr.de vantages and some disadvantages. We try to simply collect them here, without indicating a preference for one or the other.

One big advantage of Delta-k methods is that one does not have to oversample the cross-correlation function in order to estimate sub-pixel shifts. The phase difference, properly scaled with the sub-carrier difference, will directly correspond to fractional shifts. Moreover, the implementation is straightforward and computationally efficient.

Among the disadvantages we count the possibility of having to solve phase ambiguities (a typical interferometric problem) and the sensitivity to spectral shift. The impact of spectral shift depends on the bandwidth: for Delta-k methods, which work with a fraction of the total bandwidth, the spectral-shift coherence loss is a few times larger than for the full bandwidth. Depending on the case, the quality loss might be unacceptable. A solution is to shift spectrally the slave before the formation of the two subbands, so to recover coherence. In this case it is however mandatory to account for the reduction of the effective carrier separation of the two subbands, or the estimate will be biased.

\section{EARLY AND LATE MULTILOOKING}

\section{A. Definitions}

Delta-k methods require the generation of two subbands (both for master and slave) and rely on a double difference: the first difference is between master and slave (the normal interferogram), and it is done for both the lower and the upper subbands. The second difference is between the two subbands (the differential interferogram). A crucial question for the implementation is whether multilooking is done at interferogram level ("early multilooking") or at differential interferogram level ("late multilooking"). Throughout this paper, "multilooking" refers to the averaging of samples within a given window size. Using $m$ and $s$ for master and slave, and the indices 1 and 2 for the two subbands, the two possible implementations are

$$
\begin{aligned}
\xi^{\prime} & =\left(\overline{m_{1} s_{1}^{*}}\right)\left(\overline{m_{2}^{*} s_{2}}\right) \\
\xi & =\overline{m_{1} s_{1}^{*} m_{2}^{*} s_{2}} .
\end{aligned}
$$

The overbars indicate the operation of spatial averaging, and the asterisks the complex conjugation. In both cases the final step will be to take the phase of $\xi$ or $\xi^{\prime}$, e.g. $\varphi=\angle \xi$, and rescale it to a delay $(\hat{d})$, according to the spectral separation $\Delta f$ of the two subband carriers:

$$
\hat{d}=\frac{\varphi}{2 \pi \Delta f} .
$$


Before discussing the difference in the performance of the two implementations, it is worth illustrating the advantage of the "late" average implementation.

\section{B. Advantage of the "late multilooking" implementation}

The biggest advantage in using the "late" average (2) is the inherent phase compensation. If an interferometric phase is present in the averaging window, it will be (almost) the same in the upper and the lower subbands and therefore it will (almost) cancel out in the product $m_{1} s_{1}^{*} m_{2}^{*} s_{2}$. Because of this, there is no need to compensate topographic, atmospheric or deformation phase variations before averaging. Averages can actually be conducted on large areas, like is it suggested in [8]. For "early multilooking" implementations, i.e., Eq. (1), it is necessary to make sure that the phase variation within the averaging window is small enough, not to reduce the quality of the final result. The same issue affects also coherent crosscorrelation, but, obviously, not incoherent cross-correlation.

This paper will show that the advantage of "late multilooking" does not come for free but entails a significant performance loss.

\section{Performance of "Late multilooking”}

For the derivation of the performance, we will assume that the signals are distributed as complex Gaussians, with rectangular spectra, and that they are normalized such that $\mathrm{E}\left[m_{n} m_{n}^{*}\right]=\mathrm{E}\left[s_{n} s_{n}^{*}\right]=1$. We will call the coherence between master and slave $\gamma: \mathrm{E}\left[m_{1} s_{1}^{*}\right]=\mathrm{E}\left[m_{2} s_{2}^{*}\right]=\gamma$, whereas there is no correlation between the sublooks: $\mathrm{E}\left[m_{1} s_{2}^{*}\right]=$ $\mathrm{E}\left[m_{2} s_{1}^{*}\right]=\mathrm{E}\left[m_{1} m_{2}^{*}\right]=\mathrm{E}\left[s_{1} s_{2}^{*}\right]=0$.

The averaged signal or sample mean (Eq. (2)) is written explicitely as

$$
\xi=\frac{1}{4 N_{s}} \sum_{n} \xi_{n}=\frac{1}{4 N_{s}} \sum_{n} m_{1, n} s_{1, n}^{*} m_{2, n}^{*} s_{2, n},
$$

where $N_{s}$ is the number of independent samples for each subband and $\xi_{n}$ is the pixel-wise multiplication of the four subbands $\left(m_{1} s_{1}^{*} m_{2}^{*} s_{2}\right)$. To avoid aliasing, the signals are oversampled by a factor 4 before the multiplication, therefore the factor $1 / 4$ in (4).

For large $N_{s}$ the variance of the phase of $\xi$ can be approximated by the variance of the imaginary part of $\xi$, scaled by the square of the expected value of $\xi$ :

$$
\operatorname{Var}[\varphi] \approx \frac{\operatorname{Var}[\operatorname{Im}[\xi]]}{\mathrm{E}[\xi]^{2}}
$$

The variance of the imaginary part is computed as follows:

$$
\begin{aligned}
\operatorname{Var}[\operatorname{Im}[\xi]]=\mathrm{E} & {\left[\left(\frac{1}{4 N_{s}} \sum_{n} \frac{\xi_{n}-\xi_{n}^{*}}{2 i}\right)^{2}\right] } \\
=\mathrm{E} & {\left[\frac{1}{\left(4 N_{s}\right)^{2}} \sum_{n k} \frac{\xi_{n}-\xi_{n}^{*}}{2 i} \frac{\xi_{n-k}-\xi_{n-k}^{*}}{2 i}\right] } \\
=- & \frac{1}{4} \frac{1}{\left(4 N_{s}\right)^{2}} \sum_{n k} \mathrm{E}\left[\xi_{n} \xi_{n-k}-\xi_{n} \xi_{n-k}^{*}\right. \\
& \left.-\xi_{n}^{*} \xi_{n-k}+\xi_{n}^{*} \xi_{n-k}^{*}\right] .
\end{aligned}
$$

Now, assuming Gaussian speckle, it is possible to use Reed theorem [9] (also known as Wick's theorem) to compute the expected values:

$$
\begin{aligned}
& \mathrm{E}\left[\xi_{n} \xi_{n-k}\right]= \\
& =\mathrm{E}\left[m_{1, n} s_{1, n}^{*} m_{2, n}^{*} s_{2, n} m_{1, n-k} s_{1, n-k}^{*} m_{2, n-k}^{*} s_{2, n-k}\right] \\
& =\mathrm{E}\left[m_{1, n} s_{1, n}^{*} m_{1, n-k} s_{1, n-k}^{*}\right] \mathrm{E}\left[m_{2, n}^{*} s_{2, n} m_{2, n-k}^{*} s_{2, n-k}\right] \\
& =\left(\gamma^{2}+\gamma^{2} \operatorname{sinc}^{2}(k / 4)\right)^{2} \\
& \mathrm{E}\left[\xi_{n} \xi_{n-k}^{*}\right]= \\
& =\mathrm{E}\left[m_{1, n} s_{1, n}^{*} m_{2, n}^{*} s_{2, n} m_{1, n-k}^{*} s_{1, n-k} m_{2, n-k} s_{2, n-k}^{*}\right] \\
& =\mathrm{E}\left[m_{1, n} s_{1, n}^{*} m_{1, n-k}^{*} s_{1, n-k}\right] \mathrm{E}\left[m_{2, n}^{*} s_{2, n} m_{2, n-k} s_{2, n-k}^{*}\right] \\
& =\left(\gamma^{2}+\operatorname{sinc}^{2}(k / 4)\right)^{2} \\
& \mathrm{E}\left[\xi_{n}^{*} \xi_{n-k}\right]=\mathrm{E}\left[\xi_{n} \xi_{n-k}^{*}\right]=\left(\gamma^{2}+\operatorname{sinc}^{2}(k / 4)\right)^{2} \\
& \mathrm{E}\left[\xi_{n}^{*} \xi_{n-k}^{*}\right]=\mathrm{E}\left[\xi_{n} \xi_{n-k}\right]=\left(\gamma^{2}+\gamma^{2} \operatorname{sinc}^{2}(k / 4)\right)^{2} .
\end{aligned}
$$

Coming back to the variance of the imaginary part

$$
\begin{aligned}
& \operatorname{Var}[\operatorname{Im}[\xi]] \approx \\
& \approx-\frac{1}{2} \frac{1}{\left(4 N_{s}\right)^{2}} \sum_{n k}\left(\gamma^{4}\left(1+2 \operatorname{sinc}^{2}(k / 4)+\operatorname{sinc}^{4}(k / 4)\right)\right. \\
& \left.-\left(\gamma^{4}+2 \gamma^{2} \operatorname{sinc}^{2}(k / 4)+\operatorname{sinc}^{4}(k / 4)\right)\right)
\end{aligned}
$$

To proceed we extend the sums over $k$ from $-\infty$ to $+\infty$, which is a right approximation for large $N_{s}$ :

$$
\begin{aligned}
& \sum_{k} \operatorname{sinc}^{2}(k / 4)=4 \\
& \sum_{k} \operatorname{sinc}^{4}(k / 4)=8 / 3 .
\end{aligned}
$$

Thus, we have

$$
\begin{aligned}
\operatorname{Var}[\operatorname{Im}[<\xi>]] & \approx-\frac{1}{2} \frac{1}{\left(4 N_{s}\right)^{2}} \sum_{n}\left(\gamma^{4}(32 / 3)-8 \gamma^{2}-8 / 3\right) \\
& =\frac{4}{3} \frac{1}{\left(4 N_{s}\right)}\left(1+3 \gamma^{2}-4 \gamma^{4}\right) .
\end{aligned}
$$

In the last step, the sum over $n$ is substituted by a factor $4 N_{s}$, since all terms are identical.

On the other side

$$
\mathrm{E}[\xi]=\frac{1}{4 N_{s}} \sum_{n} \mathrm{E}\left[\xi_{n}\right]=\mathrm{E}\left[m_{1} s_{1}^{*} m_{2}^{*} s_{2}\right]=\gamma^{2},
$$

so that the variance of the phase of $\xi$ is approximately

$$
\operatorname{Var}[\varphi] \approx \frac{\operatorname{Var}[\operatorname{Im}[\xi]]}{\mathrm{E}[\xi]^{2}} \approx \frac{1+3 \gamma^{2}-4 \gamma^{4}}{3 N_{s} \gamma^{4}} .
$$

To get the variance of the delay estimate $\hat{d}$, one has to scale (18) by the square of the spectral separation of the two subbands (i.e., $(4 \pi / 3)^{2}$, for subbands which are $1 / 3$ of the total bandwidth) and substitute the number of independent samples if the full-bandwidth images (i.e., $N_{s}=N / 3$ ). The choice of taking the upper and lower third of the bandwith is the best compromise between subcarrier separation and number of independent samples in each subband (see [7] for a proof). 
Finally the performance of the delay estimate is:

$$
\begin{aligned}
\operatorname{Var}[\hat{d}] & \approx \frac{27}{16 \pi^{2}} \frac{1+3 \gamma^{2}-4 \gamma^{4}}{3 N \gamma^{4}} \\
& =\frac{9}{16 N} \frac{\left(1-\gamma^{2}\right)\left(1+4 \gamma^{2}\right)}{\pi^{2} \gamma^{4}}
\end{aligned}
$$

This equation is normalized to a unitary bandwidth: the square root of the variance represents shifts relative to the resolution element.

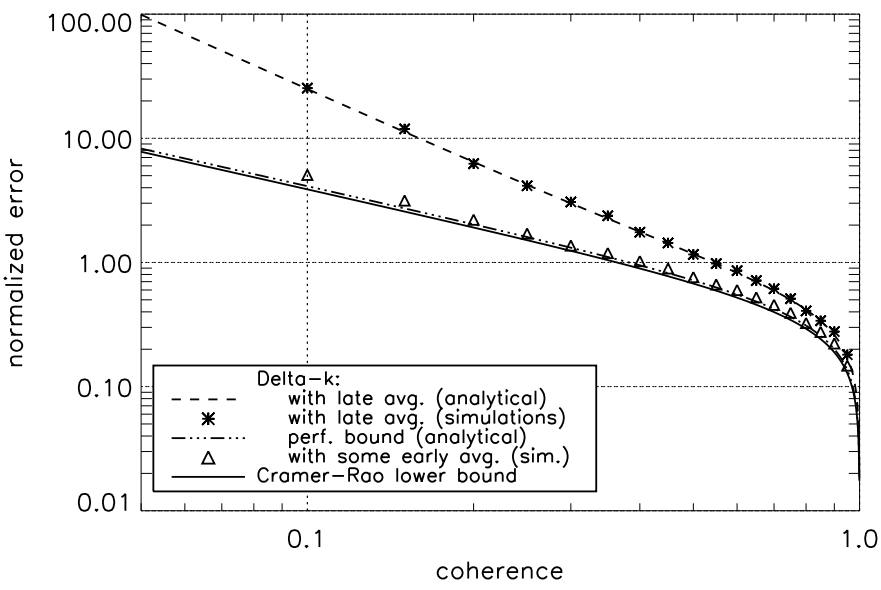

Fig. 1. The normalized accuracy $(\sigma \sqrt{N})$ of Delta-k as a function of coherence, with and without spatial averaging of the interferograms. The number of averaged independent samples in each subband is $N_{s}=5 / \gamma^{2}$ for the simulated case (triangles).

\section{COMPARISONS WITH OTHER METHODS}

The performance of "late multilooking" derived in this paper should be compared to the standard "early multilooking" and to cross-correlation techniques. It is anticipated here that the comparison will reveal unsuspected relations.

\section{A. Performance of cross-correlation methods}

We report here the asymptotic performance of Coherent Cross-Correlation [7]:

$$
\operatorname{Var}\left[\hat{d}_{\mathrm{CCC}}\right] \approx \frac{3}{2 N} \frac{1-\gamma^{2}}{\pi^{2} \gamma^{2}}=\mathrm{CRLB}
$$

and Incoherent Cross-Correlation [10]:

$$
\operatorname{Var}\left[\hat{d}_{\mathrm{ICC}}\right] \approx \frac{3}{10 N} \frac{\left(1-\gamma^{2}\right)\left(2+7 \gamma^{2}\right)}{\pi^{2} \gamma^{4}}
$$

Note that coherent cross-correlation attains asymptotically the Cramér-Rao lower bound (CRLB), as the number of independent samples tends to infinity.

\section{B. Performance of "early-multilooking"}

The asymptotic performance bound of "early-multilooking" Delta-k was derived in [7], for two subbands spanning the lower and upper third of the spectrum:

$$
\operatorname{Var}\left[\hat{d}_{\Delta k}\right] \geq \frac{27}{16 N} \frac{1-\gamma^{2}}{\pi^{2} \gamma^{2}}
$$

In that paper it was shown that its asymptotic efficiency is $8 / 9$, which means that the variance (23) is just $12.5 \%$ larger than the CRLB (21): on the plot in Fig. 1 the two are almost indistinguishable.

In terms of Fisher information this translates into a missing $1 / 9$ with respect to the information of the full spectrum. The missing information resides in each of the three subbands forming the full spectrum, taken separately, as it is shown in the following. Consider for example the middle third of the full bandwidth. The ratio between the information of the middle third and the full spectrum is $1 / 27$. A factor $1 / 9$ accounts for the resolution loss (the information goes with the inverse square of the resolution!); an additional $1 / 3$ represents the loss of independent samples, for the same data, caused by the reduced bandwidth. However, since there are three of such subbands, the contributions of the three subbands is exactly $3 \times(1 / 27)=1 / 9$.

Another way to obtain the same result is to consider a cascade of Delta-k estimators, to be applied recursively to smaller and smaller subbands. For instance, at the first level, one would apply a Delta-k on the full bandwidth (which amounts to $8 / 9$ of the total available information). Then, at the second level, one would have three Delta-k estimators, to each third of the full bandwidth, recovering $1 / 9$ of the information w.r.t. the first level (i.e. $(1 / 9) \times(8 / 9)=8 / 81)$. At the third level it is another factor $1 / 9$, and so on. Summing the geometric series shows that this infinite scheme would recover perfect efficiency: $(8 / 9) \sum_{n}(1 / 9)^{n}=1$.

We do not intend to suggested this procedure in practice, as the efficiency of $8 / 9$ is already high: it is useful in order to reconstruct theoretically the missing information and to provide an interpretation of the results in [7].

\section{Performance comparison and unexpected analogies}

It is clear that "late-multilooking" Delta-k (20) performs definitely worse than the Cramér-Rao bound (21), as one can see in Fig. 1 comparing the solid with the dashed line. For high coherences the efficiency is $3 / 5$, for low coherences it goes to zero because of the extra $\gamma^{2}$ factor. The log-scale plot reveals this fact with a clear slope difference. ${ }^{1}$ The same can be said with respect to "early-multilooking" Delta-k (23), which is very close to the CRLB. Simulations confirm the analytical derivation, as shown by the "stars" in Fig. 1.

The performance of "late multilooking" Delta-k is instead surprisingly similar to the one of incoherent cross-correlation with intensity signals derived in [10] and reported for convenience in (22). Figure 2 shows the relative efficiency between the two, which is the ratio between (20) and (22). After examining the situation more carefully, one should conclude that this is not so strange: "late-multilooking" Delta-k is totally robust to phase errors and performs like an estimator based on intensities alone. It could be seen as a frequency-domain equivalent of incoherent cross-correlation.

Since the two estimators have basically the same performance, it is natural to ask whether they are substantially the same estimator, or they have the same performance by chance.

\footnotetext{
${ }^{1}$ This visualization has been suggested by one reviewer.
} 


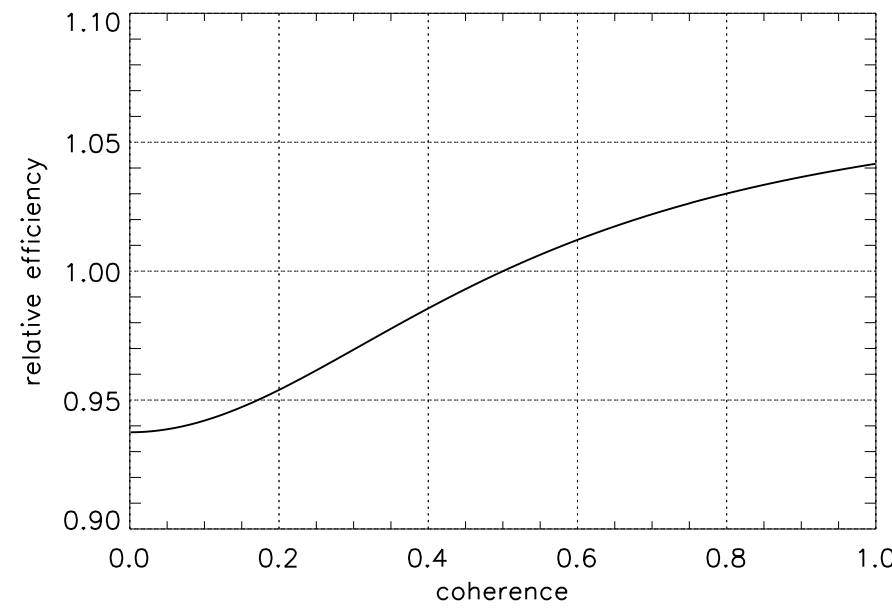

Fig. 2. The relative efficiency of "late-multilooking" Delta-k and incoherent cross-correlation as a function of coherence: the two estimators are almost equivalent.

The correlation of the shifts obtained with "late multilooking" Delta-k and incoherent cross-correlation is about 0.6-0.7 (Monte Carlo simulations), which means that they are actually quite similar without being exactly the same. Figure 3 (left) reports a cross-plot of the two estimates for 1000 simulations.
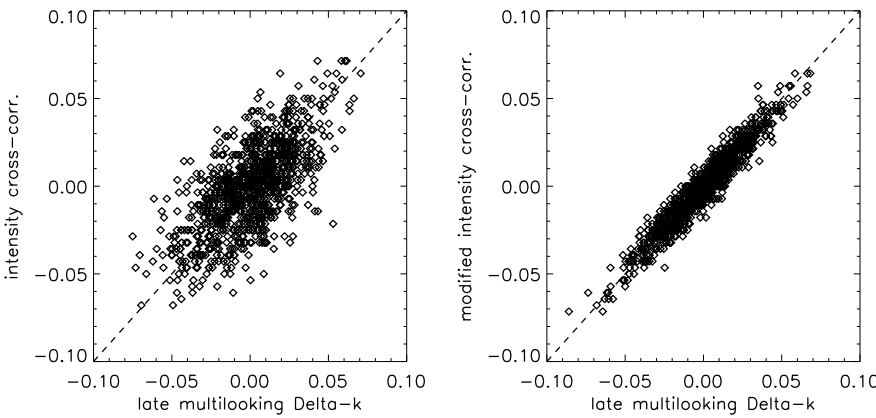

Fig. 3. Scatter plot of Monte Carlo simulations of "late multilooking" Delta-k and cross-correlation of intensities. Left: intensities of full-spectrum signals. Right: intensities of filtered signals. The correlation between the two estimators is 0.68 on the left and 0.96 on the right.

\section{Equalization of intensity spectra}

The fact that the two estimators are not totally correlated leaves room for some improvement, for example averaging the results of the two. The advantage is anyway rather small, about $1 \mathrm{~dB}$.

The explanation of the small difference is that the two estimators are essentially working on the same signals (the intensities), but with different spectral weightings. Incoherent cross-correlation takes the full spectrum of the intensity signal, which is shaped like a triangle, thus privileging low frequencies. "Late multilooking" Delta-k is sensitive to the high frequencies that result from computing the intensities only with the subbands signals. The average of the two is analogous to working with a whitened intensity spectrum, which maximizes both the number of independent samples and the resolution.
This interpretation is reinforced by the following observation: if the original signals are pre-filtered to remove the central third of the spectrum, an operation that does not affect Delta-k estimates, the correlation between intensity crosscorrelation and "late-multilooking" Delta-k raises towards unity, as one can see in Figure 3 (right).

\section{EARLY-LATE COMPROMISE}

Of course it is not necessary to choose between doing all the averaging at interferogram level or doing it only after the differential interferogram formation. The recommended compromise will be to perform a sufficient amount of averaging at interferogram level, leaving the rest after the formation of the differential interferogram. The question is: how much early multilooking is actually needed? The answer depends on the coherence, with low coherence interferograms requiring more "early multilooking" for the same efficiency threshold.

The question of Delta-k efficiency goes really back to the efficiency of the maximum-likelihood interferometric phase estimator [11]. Almost constant efficiency levels are obtained for a number of independent samples $N$ that satisfies the equation $\gamma^{2} N=N_{0}$ (const.). Figure 4 reports the efficiency of the maximum-likelihood phase estimator (for complex Gaussian signals) for different $N_{0}$ levels. With $N_{0}=4$ or 5 the efficiency is already quite high $(0.8-0.85)$.

Figure 1 reports also the performance of Delta-k shift estimation with $N_{s}=5 / \gamma^{2}$ independent samples averaged at interferogram level. This is enough to achieve a reasonable efficiency according to the simulations (triangles). Note also that in most practical cases the phase is flattened with external information (DEM or ellipsoid), so that a relatively large "early multilooking" might be acceptable, depending on the quality of the DEM and the height of ambiguity.

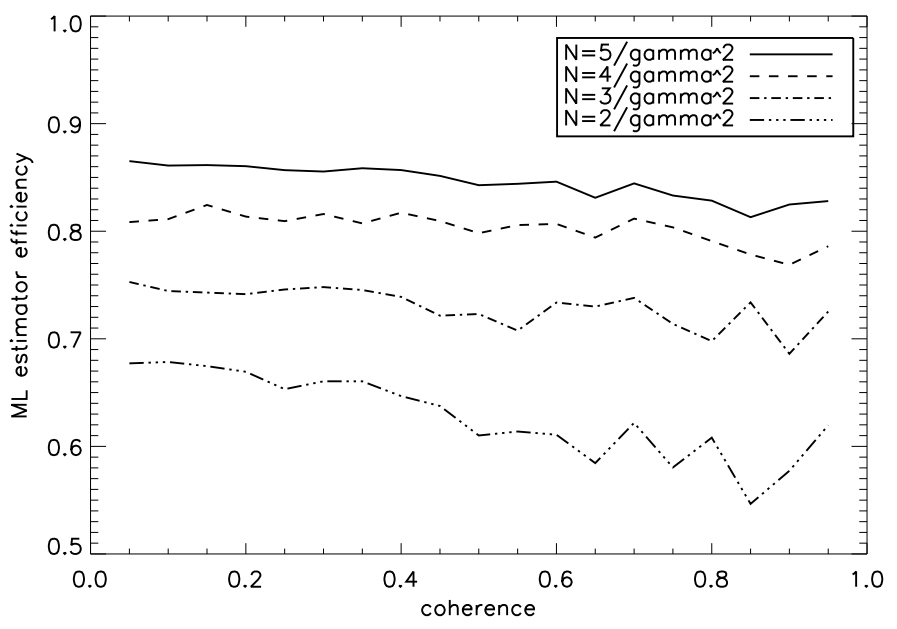

Fig. 4. The efficiency of the maximum-likelihood estimator of the interferometric phase for Gaussian signals, as a function of coherence and with $N_{0} / \gamma^{2}$ independent samples, for different $N_{0}$.

The second stage averaging poses an additional challenge. Because of backscatter variations it might not be advisable to retain the amplitude information, so that one might want to normalize the differential interferogram before further averaging. Working directly with phases or shifts would also 
be a possibility, provided that one takes care of possible ambiguities, i.e., phase wrapping in the differential phase.

\section{CONCLUSION}

This letter has discussed the performance of Delta-k estimators. The presence of uncompensated fringes in the averaging window calls for a late-multilooking strategy, which intrinsically removes interferometric phases. However we have shown that, to preserve the estimator efficiency, it is mandatory to performs a certain amount of early averaging at interferogram level, according to the coherence level. The pure late-multilooking Delta-k estimator is totally insensitive to phase errors, but its performance is closer to incoherent crosscorrelation rather than coherent cross-correlation. Theory and simulations agree with each other and confirm the finding.

\section{AKNOWLEDGMENT}

FDZ would like to acknowledge the valuable comments and suggestions of R. Bamler, G. Gomba, F. Rocca and the anonymous reviewers.

\section{REFERENCES}

[1] S. Madsen and H. Zebker, "Topographic mapping using radar interferometry: Processing techniques," Geoscience and Remote Sensing, IEEE Transactions on, vol. 31, pp. 246-256, Jan 1993.

[2] R. Scheiber and A. Moreira, "Coregistration of interferometric SAR images using spectral diversity," Geoscience and Remote Sensing, IEEE Transactions on, vol. 38, no. 5, pp. 2179 -2191, Sep 2000.

[3] P. Prats and J. Mallorqui, "Estimation of phase undulations with multisquint processing in airborne interferometric SAR images," Geoscience and Remote Sensing, IEEE Transactions on, vol. 41, no. 6, pp. 15301533, Jun 2003.

[4] A. Reigber, P. Prats, and J. Mallorqui, "Refined estimation of timevarying baseline errors in airborne SAR interferometry," Geoscience and Remote Sensing Letters, IEEE, vol. 3, no. 1, pp. 145-149, Jan 2006.

[5] M. Rodriguez-Cassola, P. Prats-Iraola, P. Lopez-Dekker, A. Reigber, G. Krieger, and A. Moreira, "Autonomous time and phase calibration of spaceborne bistatic SAR systems," in EUSAR 2014; 10th European Conference on Synthetic Aperture Radar; Proceedings of, June 2014, pp. 264-267.

[6] S. Tebaldini, A. Monti Guarnieri, and F. Rocca, "Recovering time and space varying phase screens through SAR multi-squint differential interferometry," in Synthetic Aperture Radar, 2012. EUSAR. 9th European Conference on, April 2012, pp. 16-19.

[7] R. Bamler and M. Eineder, "Accuracy of differential shift estimation by correlation and split-bandwidth interferometry for wideband and Delta-k SAR systems," IEEE Geosci. Remote Sensing Letters, vol. 2, no. 2, pp. 151-155, Apr 2005.

[8] P. Prats-Iraola, R. Scheiber, L. Marotti, S. Wollstadt, and A. Reigber, "TOPS interferometry with TerraSAR-X," Geoscience and Remote Sensing, IEEE Transactions on, vol. 50, no. 8, pp. 3179-3188, Aug 2012.

[9] I. Reed, "On a moment theorem for complex Gaussian processes," Information Theory, IRE Transactions on, vol. 8, no. 3, pp. $194-195$, Apr 1962.

[10] F. De Zan, "Accuracy of incoherent speckle tracking for circular Gaussian signals," Geoscience and Remote Sensing Letters, IEEE, vol. 11, no. 1, pp. 264-267, Jan 2014.

[11] M. Seymour and I. Cumming, "Maximum likelihood estimation for SAR interferometry," in International Geoscience and Remote Sensing Symposium, 1994., vol. 4, Aug 1994, pp. 2272-2275. 